\title{
Estimation of genetic variability, GCA and SCA effects for development of early and high yielding bell pepper hybrids suitable for protected cultivation
}

\author{
Mayanglambam Bilashini Devi ${ }^{*}$, N.K. Pathania ${ }^{2}$ and Nisha Thakur ${ }^{3}$ \\ ${ }^{1}$ Division of Horticulture, ICAR-Research Complex for NEH Region, Umiam (Meghalaya), INDIA \\ ${ }^{2}$ CSK Himachal Pradesh Krishi Vishvavidhyalaya, Palampur-176062 (HP), INDIA \\ ${ }^{3}$ ICAR-IARI Regional Station, Katrain, Kullu Valley-175129 (HP), INDIA \\ *Corresponding author. E-mail: bilashini1712@gmail.com \\ Received: October 31, 2017; Revised received: November 16, 2017; Accepted: February 13, 2018
}

\begin{abstract}
The present investigation involves 29 diverse bell pepper genotypes for variability studies and 14 promising lines (11 lines and 3 testers), $33 F_{1}$ and 1 standard check for combining ability studies. The objective of the study was to assess the nature and magnitude of genetic variability among the bell pepper genotypes for yield and other horticultural traits and to identify the potential parental lines and cross combinations suitable for the protected environment based on the estimation of general combining ability and specific combining ability. A wide range of variability was observed for most of the characters studied. The genotypes viz., Kashmir Sel-1 ( $\left.L_{2}\right)$, DARL-10 ( $\left.L_{9}\right)$ and $\operatorname{UHFSP}(Y)-11\left(L_{11}\right)$ for marketable fruit yield per plant and Kashmir Sel-1 $\left(L_{2}\right)$, UHFSP $(Y)-11\left(L_{11}\right)$ and DARL-01 $\left(L_{5}\right)$ for earliness were good general combiners and these may be included for exploiting hybrid vigour or development of purelines. On the basis of specific combining ability effects and per se performance, the crosses viz., $A R C H-19\left(L_{1}\right)$ $\times$ Solan Bharpur $\left(T_{1}\right)$, DARL-10 $\left(L_{9}\right) \times$ California Wonder $\left(T_{2}\right)$ and UHFSP $(Y)-11\left(L_{11}\right) \times$ Yolo Wonder $\left(T_{3}\right)$ were the best cross combinations for marketable fruit yield per plant and number of marketable fruits per plant under protected environment. The cross combinations viz., DARL-10 $\left(\mathrm{L}_{9}\right) \times$ California Wonder $\left(\mathrm{T}_{2}\right)$, DARL-01 $\left(\mathrm{L}_{5}\right) \times \mathrm{California}$ Wonder $\left(T_{2}\right)$, LC $\left(L_{10}\right) \times$ Solan Bharpur $\left(T_{1}\right)$ and Kandaghat Sel-9 $\left(L_{7}\right) \times$ Solan Bharpur $\left(T_{1}\right)$ shows significant negative SCA for earliness and could be used for development of early hybrids.
\end{abstract}

Keywords: Capsicum annuum L. var. grossum Sendt., Combining ability, Protected environment, Mid Hill, Western Himalayas

\section{INTRODUCTION}

Bell pepper (Capsicum annuum var. grossum Sendt.) is one of the most popular and highly remunerative annual herbaceous vegetable crops cultivated extensively in India. It holds a very prominent position as the leading off-season vegetable generating cash revenue to the farmers of Western Himalayas by selling the produce in the neighbouring states and metropolitan cities. It is commercially cultivated as a cash crop during June to October in mid hills of Western Himalayas. Because of its economic importance as a high value vegetable crop, both in domestic and overseas markets quality production of capsicum is the need of the day. This has led to the production of capsicum under protected conditions to meet the standards of different markets prompting popularity of this crop as an enterprise in urban and peri urban areas. Moreover, the supply is inadequate due to the low productivity of the crop as the crop grown under open conditions does not fulfill the urban market standards due to the prevalence of various biotic (pests and diseases) and abiotic (heavy rains, low and high temperature and high relative humidity) stress. In this context, growing of bell pepper under protected structures will ensure promise for higher yield with better quality produce. Systematic efforts to identify the promising lines and develop location specific hybrids of bell pepper for protected cultivation are limited. Therefore, there is a need to assess the available germplasm of bell pepper for genetic variability under protected environment and also study the combining ability among promising lines with the perspective of identifying the best performing and location specific hybrids suitable for cultivation under protected environment in mid hills of Western Himalayas

Genetic improvement of yield and its contributing characters require selection of appropriate breeding procedures, which are largely dependent upon the study of general combining ability (GCA) of parents and specific combining ability (SCA) of hybrids ( Sahoo and Singh 2017). Information on combining ability will facilitate the choice of suitable parents for 
hybridization program to develop $F_{1}$ hybrids. Amongst the various methods to estimate combining ability effects, the line $\times$ tester mating design gives a fairly good idea of both general and specific combining abilities of parents and hybrids, respectively. Combining ability also indicates the nature and magnitude of gene action involved in the expression of quantitative traits (Kumar and Kumar, 2017). The knowledge of nature and magnitude of gene action controlling the inheritance of yield and its contributing traits, along with, proportional contribution of parental lines in the expression of different traits in $\mathrm{F}_{1}$ hybrids, would facilitate choice of efficient breeding method and suitable parental lines/testers for genetic improvement of any crop (Rattan and Chadha 2009, Kumar and Kumar 2017). In literature, sufficient information on genetic variability and combining ability studies in capsicum under open environment are available but, those involving protected environment are limited. Based upon all these considerations, the present investigation was planned and executed.

\section{MATERIALS AND METHODS}

The experimental material for the present study comprised of 29 genotypes of bell pepper for genetic variability studies during summer-autumn of $2012, F_{1}$ population of 33 crosses which was developed by crossing 14 promising genotypes (11 lines and 3 testers) selected from 29 genotypes evaluated during 2012. The crosses were made as per line $\times$ tester mating design. The $F_{1}$ population of 33 crosses along with parents (11 lines and 3 testers) and standard check (Indra) were evaluated during summerautumn, 2013 in modified naturally ventilated polyhouse at the Experimental Farm, Department of Vegetable Science and Floriculture which is situated at $32^{\circ} 6^{\prime} \mathrm{N}$ latitude and $76^{\circ} 3$ ' E longitude at an elevation of $1290.8 \mathrm{~m}$ above mean sea level with East-West orientation. The polyhouse is equipped with essential features like double door, side and top ventilation, drip and fogging facility and shading with 50 per cent green agro UV stabilized shade net. Healthy seedlings were raised in plug trays inside growth chamber. The experiment was laid out in randomized block design (RBD) with three replications. The seedlings were transplanted on $20 \mathrm{~cm}$ raised bed having 90 $\mathrm{cm}$ width. Each bed consisted of two rows of $1.5 \mathrm{~m}$ length accommodating ten plants per genotype. The plants were spaced at $45 \times 30 \mathrm{~cm}$ inter and intra row spacings. All the recommended agronomic practices and plant protection measures were followed to raise a healthy crop. The observations were recorded on randomly taken five competitive plants of each genotype in each replication for the traits viz., days to 50 per cent flowering, days to first harvest, number of marketable fruits per plant, pericarp thickness $(\mathrm{mm})$, lobes per fruit, fruit length $(\mathrm{cm})$, fruit width $(\mathrm{cm})$, average fruit weight $(\mathrm{g})$, marketable fruit yield per plant $(\mathrm{kg})$, plant height $(\mathrm{cm})$, harvest duration (days), ascorbic acid content $(\mathrm{mg} / 100 \mathrm{~g})$ and capsaicin content (\%) during both the years. The Ascorbic acid content was estimated at marketable green fruit stage by 2, 6- dichlorophenol-indophenol Visual Titration Method as described by Ranganna (1979). The capsaicin content in the marketable green fruits was determined by Colorimetric method using Folin-Ciocalteau reagent.

Statistical analysis: The genotypic, phenotypic coefficients of variations and heritability were estimated as per the method of Burton and Devane 1953. Genetic advance (GA) was calculated as per Burton and Devane 1953 and Johnson et al. 1955. The statistical analysis for combining ability studies (line $\times$ tester) was carried out as per the model suggested by Kempthrone 1957.

The parameters of variability were estimated following Burton and Devane 1953. The limits used for categorizing the magnitude of different parameters are mentioned in Table 1.

\section{RESULTS AND DISCUSSION}

Variability studies: Analysis of variance revealed significant differences among the genotypes for all the traits studied during 2012 (Table 2). The extent of variability for yield and horticultural traits of bell pepper in terms of phenotypic coefficient of variation (PCV), genotypic coefficient of variation (GCV) along with heritability $\left(\mathrm{h}^{2}\right)$ and genetic advance (GA) during 2012 are presented in Table 3. Results obtained from the present investigation on genetic variability studies during summer-autumn, 2012, revealed relatively low differences between the genotypic and phenotypic variances for all the traits studied. This indicated highly heritable and comparatively stable nature of the characters and thus, the selection based on phenotypic performance would be quite effective in the improvement of these traits. According to the classification given in Table 1 high estimates of PCV and GCV were observed for number of marketable fruits per plant $(34.81$ $\%, 32.50 \%$ ), marketable fruit yield per plant $(31.15 \%$, $29.78 \%)$, ascorbic acid content $(25.98 \%, 25.26 \%)$ and capsaicin content $(28.55 \%, 25.71 \%)$ suggesting substantial variability for the traits thereby ensuring ample scope for improvement of these traits through selection. The exploration of genetic variability in available germplasm is a pre-requisite for identification and development of new improved high yielding genotypes to further boost up the yield potential of the crop. The possibility of improvement in any crop is measured by the variability available in the crop. In general, high PCV and GCV indicate that there is a scope for selection and improvement of the particular

Table 1. Categorization of parameters of variability.

\begin{tabular}{llll}
\hline Component & High (\%) & Moderate (\%) & Low (\%) \\
\hline GCV and PCV & $>20$ & $10-20$ & $<10$ \\
Heritability & $>80$ & $60-80$ & $<60$ \\
Genetic advance & $>30$ & $15-30$ & $<15$ \\
\hline
\end{tabular}


Table 2. Analysis of variance for yield and horticultural traits in bell pepper during 2012.

\begin{tabular}{|c|c|c|c|c|}
\hline \multirow{3}{*}{ Traits } & \multicolumn{4}{|c|}{ Mean sum of squares } \\
\hline & Source & Replication & Genotype & Error \\
\hline & df & 2 & 28 & 56 \\
\hline Days to $50 \%$ flowering & & 12.63 & $24.71 *$ & 2.56 \\
\hline Days to first harvest & & 64.22 & $30.61^{*}$ & 17.14 \\
\hline Number of marketable fruits per plant & & 0.47 & $181.18^{*}$ & 8.46 \\
\hline Pericarp thickness (mm) & & 0.06 & $1.07 *$ & 0.21 \\
\hline Lobes per fruit & & 0.09 & $0.90 *$ & 0.11 \\
\hline Fruit length $(\mathrm{cm})$ & & 2.81 & $3.50 *$ & 0.64 \\
\hline Fruit width $(\mathrm{cm})$ & & 12.59 & $1.85^{*}$ & 0.42 \\
\hline Average fruit weight $(\mathrm{g})$ & & 44.69 & $152.12^{*}$ & 26.04 \\
\hline Marketable fruit yield per plant $(\mathrm{kg})$ & & 0.02 & $0.31 *$ & 0.01 \\
\hline Plant height $(\mathrm{cm})$ & & 249.12 & $2838.61 *$ & 406.50 \\
\hline Harvest duration (days) & & 499.69 & $267.52 *$ & 125.13 \\
\hline Ascorbic acid content (mg per $100 \mathrm{~g}$ ) & & 76.45 & $873.80 *$ & 9.37 \\
\hline Capsaicin content $(\%)$ & & 0.0002 & $0.01 *$ & 0.0004 \\
\hline
\end{tabular}

*Significant at $\mathrm{P}=0.05 ; \quad \mathrm{df}=$ degree of freedom

Table 3. Estimates of variability parameters for yield and horticultural traits in bell pepper during 2012.

\begin{tabular}{|c|c|c|c|c|c|}
\hline \multirow{2}{*}{ Traits } & \multicolumn{3}{|c|}{ Coefficient of variation (\%) } & \multirow{2}{*}{$\begin{array}{l}\text { Heritability } \\
(\%)\left(\mathrm{h}^{2}{ }\right)\end{array}$} & \multirow{2}{*}{$\begin{array}{l}\text { Genetic advance } \\
\text { (\% of mean) }\end{array}$} \\
\hline & Phenotypic & Genotypic & Environmental & & \\
\hline Days to $50 \%$ flowering & 7.49 & 6.46 & 3.80 & 74.20 & 11.46 \\
\hline Days to first harvest & 6.03 & 2.75 & 5.36 & 20.80 & 2.58 \\
\hline Number of marketable fruits per plant & 34.81 & 32.50 & 12.46 & 87.20 & 62.52 \\
\hline Pericarp thickness (mm) & 21.57 & 16.45 & 13.96 & 58.10 & 25.83 \\
\hline Lobes per fruit & 20.71 & 17.44 & 11.16 & 70.90 & 30.26 \\
\hline Fruit length $(\mathrm{cm})$ & 18.78 & 14.55 & 11.87 & 60.00 & 23.23 \\
\hline Fruit width $(\mathrm{cm})$ & 17.73 & 12.94 & 12.12 & 53.30 & 19.45 \\
\hline Average fruit weight $(\mathrm{g})$ & 17.38 & 13.66 & 10.75 & 61.70 & 22.11 \\
\hline Marketable fruit yield per plant $(\mathrm{kg})$ & 31.15 & 29.78 & 9.14 & 91.40 & 58.64 \\
\hline Plant height $(\mathrm{cm})$ & 21.08 & 17.20 & 12.18 & 66.60 & 28.92 \\
\hline Harvest duration (days) & 11.69 & 6.13 & 9.95 & 27.50 & 7.44 \\
\hline Ascorbic acid content (mg per $100 \mathrm{~g}$ ) & 25.98 & 25.26 & 4.61 & 96.90 & 34.41 \\
\hline Capsaicin content $(\%)$ & 28.55 & 25.71 & 12.40 & 81.10 & 0.08 \\
\hline
\end{tabular}

Table 4. Analysis of variance for combining ability for yield and horticultural traits in bell pepper during 2013.

\begin{tabular}{|c|c|c|c|c|c|c|}
\hline Source of variation & & Replication & Line & Tester & line $\times$ tester & Error \\
\hline Traits & df & 2 & 10 & 2 & 20 & 64 \\
\hline Days to $50 \%$ flowering & & 4.03 & $106.78^{* \neq}$ & $143.12^{* \neq}$ & $99.48^{*}$ & 1.52 \\
\hline Days to first harvest & & 1.40 & 115.73 & $145.49^{* \neq}$ & $133.75^{*}$ & 1.50 \\
\hline Number of marketable fruits per plant & & 5.35 & $150.48^{* \neq}$ & $143.84^{* \neq}$ & $71.58 *$ & 4.64 \\
\hline Pericarp thickness (mm) & & 0.02 & $1.75^{* \neq}$ & $5.16^{* \neq}$ & $1.26^{*}$ & 0.18 \\
\hline Lobes per fruit & & 0.08 & 0.16 & 0.06 & $0.25^{*}$ & 0.12 \\
\hline Fruit length $(\mathrm{cm})$ & & 0.47 & $1.75^{* \neq}$ & $5.91 * \neq$ & $1.24 *$ & 0.23 \\
\hline Fruit width $(\mathrm{cm})$ & & 0.38 & 0.56 & $1.10^{* \neq}$ & $0.64^{*}$ & 0.26 \\
\hline Average fruit weight $(\mathrm{g})$ & & 27.96 & $173.83^{* \neq}$ & $190.02 * \neq$ & $113.24 *$ & 26.25 \\
\hline Marketable fruit yield per plant $(\mathrm{kg})$ & & 0.01 & $0.30^{* \neq}$ & $0.22 * \neq$ & $0.12 *$ & 0.01 \\
\hline Plant height $(\mathrm{cm})$ & & 11.82 & $2499.36^{* \neq}$ & 1526.61 & $1850.71 *$ & 16.90 \\
\hline Harvest duration (days) & & 17109.29 & $834.22 * \neq$ & $480.21^{* \neq}$ & $354.57 *$ & 222.10 \\
\hline Ascorbic acid content (mg per $100 \mathrm{~g}$ ) & & 23.36 & $1622.40^{* \neq}$ & $3705.63 * \neq$ & $753.49 *$ & 37.20 \\
\hline Capsaicin content $(\%)$ & & 0.0003 & $0.007 * \neq$ & 0.0001 & $0.004 *$ & 0.0003 \\
\hline
\end{tabular}

* Significant at $5 \%$ level of significance when tested against mean squares due to error, ${ }^{\#}$ Significant at $5 \%$ level of significance when tested against mean squares due to line $\times$ tester

traits (Abebe, 2017). High estimates of PCV and GCV have also been reported by earlier workers viz. Islam and Singh, 2006 and Kumari, 2013 for yield per plant and number of fruits per plant; Sood et al., 2007 for marketable fruit yield per plant and capsaicin content and for marketable fruit yield per plant and ascor- bic acid content thereby, supporting the present findings. High PCV and moderate GCV values for pericarp thickness $(21.57 \%, 16.45 \%)$, lobes per fruit ( $20.71 \%, 17.44 \%$ ) and plant height ( $21.08 \%, 17.20$ $\%$ while, moderate PCV and GCV for fruit length $(18.78 \%, 14.55 \%)$, fruit width $(17.73 \%, 12.94 \%)$ 


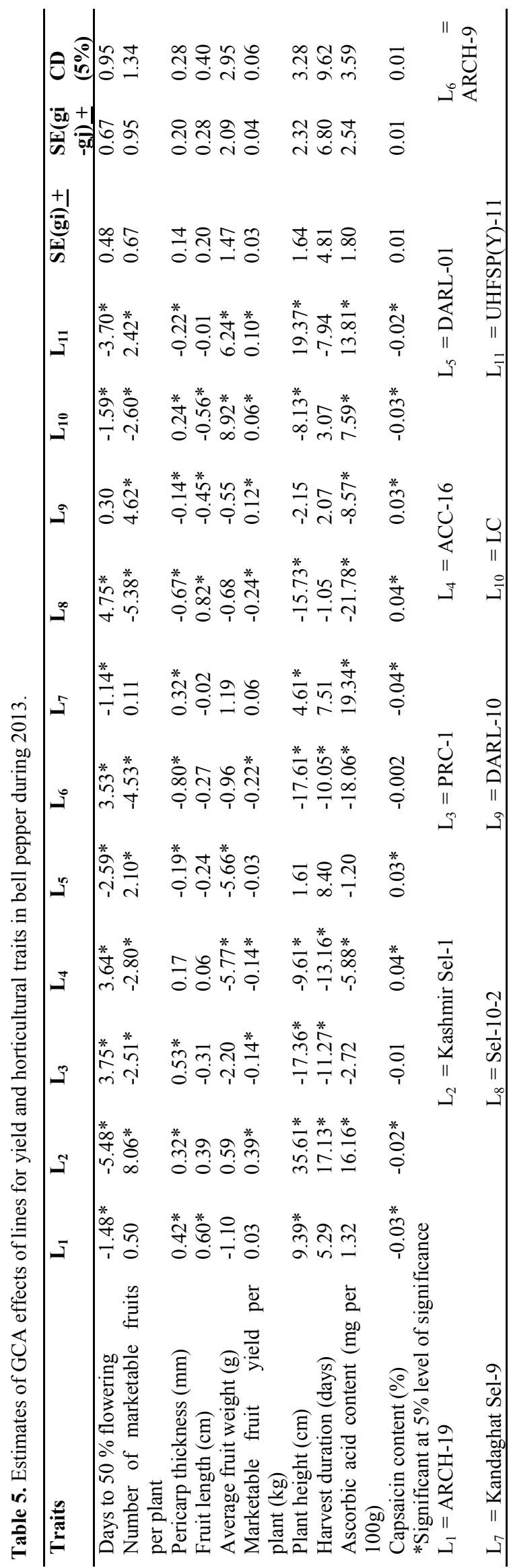

and average fruit weight $(17.38 \%, 13.66 \%)$ were observed. High PCV followed by moderate GCV for pericarp thickness, lobes per fruit and plant height revealed considerable environmental effect in the expression of these traits. Afroza et al., 2013 have also reported moderate estimates of GCV for fruit length, fruit diameter, flesh thickness, average fruit weight and number of fruits per plant. The deviation in the result obtained could be attributed to the differences in experimental material used and growing environment. Moderate to low PCV coupled with low GCV for the characters viz., harvest duration, days to 50 per cent flowering and days to first harvest indicated that these characters are largely governed by non-genetic factors. Hence, the genetic improvement of these traits through simple selection procedures appears to be quite difficult.

High heritability coupled with high genetic advance as per cent of mean were observed for ascorbic acid content $(96.90 \%, 34.41 \%)$, marketable fruit yield per plant $(91.40 \%, 58.64 \%)$ and number of marketable fruits per plant $(87.20 \%, 62.52 \%)$, indicated the presence of additive effects for these characters. Therefore, a high genetic gain can be anticipated by selection procedure.

Combining ability studies: The success of any plant breeding programme greatly depends on the right choice of parents. The potential of variety is judged by comparing the mean performance and combining the ability of the parents (Singh 1993). Kadambavanasundaram (1980) suggested that the parents with high per se performance may not always be able to transmit their superior traits into hybrids and so assessment of combining ability is most needed. General combining ability (GCA) of a parent is a factor that predicts the performance of a parent over a series of cross combinations (Rohini et al., 2017).

The analysis of variance for combining ability revealed significant differences among crosses for all the traits studied (Table 4). Mean squares due to lines were significant for all the traits except days to first harvest, lobes per fruit and fruit width, when tested against both mean squares due to error and line $\times$ tester interactions. The testers exhibited significant differences for all the traits except lobes per fruit, plant height and capsaicin content when tested against both mean squares due to error and line $\times$ tester interactions. Mean squares due to line $\times$ tester interactions were significant for all the traits studied. This indicated the presence of considerable genetic diversity among the parents (lines and testers) used in the present study.

The line, Kashmir Sel-1 $\left(\mathrm{L}_{2}\right)$ was the good general combiner for earliness followed by the lines, UHFSP (Y)-11 $\left(\mathrm{L}_{11}\right)$, DARL-01 $\left(\mathrm{L}_{5}\right)$, LC $\left(\mathrm{L}_{10}\right)$, ARCH-19 $\left(\mathrm{L}_{1}\right)$ and Kandaghat Sel-9 $\left(\mathrm{L}_{7}\right)$ as these exhibited significant negative general combining ability ef- 
Mayanglambam Bilashini Devi et al. / J. Appl. \& Nat. Sci. 10 (1): 410 - 416 (2018)

Table 6. Estimates of GCA effects of testers for yield and horticultural traits in bell pepper during 2013.

\begin{tabular}{|c|c|c|c|c|c|c|}
\hline Traits & $T_{1}$ & $\mathbf{T}_{2}$ & $\mathbf{T}_{3}$ & $\mathrm{SE}(\mathrm{gi}) \pm$ & SE $($ gi-gj $) \pm$ & $\mathrm{CD}(5 \%)$ \\
\hline Days to $50 \%$ flowering & $-2.39 *$ & $1.39 *$ & $1.00^{*}$ & 0.25 & 0.35 & 0.50 \\
\hline Days to first harvest & $-2.40 *$ & $0.93 *$ & $1.48 *$ & 0.24 & 0.34 & 0.49 \\
\hline Number of marketable fruits per plant & $1.83 *$ & 0.45 & $-2.28 *$ & 0.35 & 0.50 & 0.70 \\
\hline Pericarp thickness (mm) & $0.33 *$ & 0.11 & $-0.44 *$ & 0.07 & 0.10 & 0.15 \\
\hline Fruit length (cm) & $-0.30 *$ & -0.19 & $0.49 *$ & 0.10 & 0.15 & 0.21 \\
\hline Fruit width $(\mathrm{cm})$ & -0.10 & -0.11 & $0.21 *$ & 0.09 & 0.14 & 0.19 \\
\hline Average fruit weight $(\mathrm{g})$ & -0.45 & $-2.15^{*}$ & $2.59^{*}$ & 0.77 & 1.09 & 1.54 \\
\hline Marketable fruit yield per plant $(\mathrm{kg})$ & $0.09 *$ & $-0.04 *$ & $-0.05^{*}$ & 0.02 & 0.04 & 0.03 \\
\hline Harvest duration (days) & $4.27 *$ & -3.08 & -1.19 & 2.15 & 3.56 & 5.02 \\
\hline Ascorbic acid content (mg per 100g) & 0.87 & $10.14 *$ & $-11.01 *$ & 0.94 & 1.33 & 1.88 \\
\hline
\end{tabular}

*Significant at $5 \%$ level of significance, $\mathrm{T}_{1}=$ Solan Bharpur, $\mathrm{T}_{2}=$ California Wonder, $\mathrm{T}_{3}=$ Yolo Wonder

Table 7. Top three crosses with significant SCA and GCA effects for yield and horticultural traits in bell pepper.

\begin{tabular}{|c|c|c|c|c|}
\hline Traits & Crosses with significant SCA effects & $\begin{array}{l}\begin{array}{l}\text { SCA } \\
\text { effects }\end{array} \\
\end{array}$ & $\begin{array}{l}\text { per se per- } \\
\text { formance }\end{array}$ & GCA effects \\
\hline \multirow{3}{*}{ Days to $50 \%$ flowering } & DARL-10 $\left(\mathrm{L}_{9}\right) \times$ California Wonder $\left(\mathrm{T}_{2}\right)$ & $-9.39 *$ & 47.00 & average $\times$ good \\
\hline & DARL-01 $\left(\mathrm{L}_{5}\right) \times$ California Wonder $\left(\mathrm{T}_{2}\right)$ & $-9.17 *$ & 31.33 & good $\times$ poor \\
\hline & LC $\left(\mathrm{L}_{10}\right) \times$ Solan Bharpur $\left(\mathrm{T}_{1}\right)$ & $-7.72 *$ & 30.00 & good $\times$ good \\
\hline \multirow[t]{3}{*}{ Days to first harvest } & DARL-10 $\left(\mathrm{L}_{9}\right) \times$ California Wonder $\left(\mathrm{T}_{2}\right)$ & $-13.71 *$ & 50.67 & average $\times$ poor \\
\hline & LC $\left(\mathrm{L}_{10}\right) \times$ Solan Bharpur $\left(\mathrm{T}_{1}\right)$ & $-9.60^{*}$ & 51.67 & average $\times$ good \\
\hline & DARL-01 $\left(\mathrm{L}_{5}\right) \times$ California Wonder $\left(\mathrm{T}_{2}\right)$ & $-8.60^{*}$ & 56.67 & average $\times$ poor \\
\hline \multirow[t]{3}{*}{ Number of marketable fruits/plant } & DARL-10 $\left(\mathrm{L}_{9}\right) \times$ California Wonder $\left(\mathrm{T}_{2}\right)$ & $10.03 *$ & 37.00 & good $\times$ average \\
\hline & $\operatorname{UHFSP}(\mathrm{Y})-11\left(\mathrm{~L}_{11}\right) \times$ Yolo Wonder $\left(\mathrm{T}_{3}\right)$ & $9.40^{*}$ & 31.45 & good $\times$ poor \\
\hline & ARCH-19 $\left(\mathrm{L}_{1}\right) \times$ Solan Bharpur $\left(\mathrm{T}_{1}\right)$ & $4.40 *$ & 28.63 & average $\times$ good \\
\hline \multirow[t]{3}{*}{ Pericarp thickness (mm) } & $\operatorname{UHFSP}(\mathrm{Y})-11\left(\mathrm{~L}_{11}\right) \times$ Yolo Wonder $\left(\mathrm{T}_{3}\right)$ & $1.25 *$ & 3.90 & poor $\times$ poor \\
\hline & DARL-10 $\left(\mathrm{L}_{9}\right) \times$ California Wonder $\left(\mathrm{T}_{2}\right)$ & $1.10^{*}$ & 4.38 & poor $\times$ average \\
\hline & ARCH-19 $\left(\mathrm{L}_{1}\right) \times$ Solan Bharpur $\left(\mathrm{T}_{1}\right)$ & $0.64 *$ & 4.70 & $\operatorname{good} \times \operatorname{good}$ \\
\hline Lobes per fruit & $\operatorname{UHFSP}(\mathrm{Y})-11\left(\mathrm{~L}_{11}\right) \times$ Yolo Wonder $\left(\mathrm{T}_{3}\right)$ & $0.51 *$ & 3.80 & average $\times$ average \\
\hline \multirow[t]{3}{*}{ Fruit length $(\mathrm{cm})$} & Kandaghat Sel-9 $\left(\mathrm{L}_{7}\right) \times$ Yolo Wonder $\left(\mathrm{T}_{3}\right)$ & $0.94^{*}$ & 7.75 & average $\times$ good \\
\hline & ACC-16 $\left(\mathrm{L}_{4}\right) \times$ Solan Bharpur $\left(\mathrm{T}_{1}\right)$ & $0.71 *$ & 6.81 & average $\times$ poor \\
\hline & DARL-10 $\left(\mathrm{L}_{9}\right) \times$ California Wonder $\left(\mathrm{T}_{2}\right)$ & $0.71 *$ & 6.41 & poor $\times$ average \\
\hline Fruit width $(\mathrm{cm})$ & $\operatorname{UHFSP}(\mathrm{Y})-11\left(\mathrm{~L}_{11}\right) \times$ Yolo Wonder $\left(\mathrm{T}_{3}\right)$ & $0.73 *$ & 6.23 & average $\times$ good \\
\hline \multirow{3}{*}{ Average fruit weight (g) } & DARL-10 $\left(\mathrm{L}_{9}\right) \times$ Yolo Wonder $\left(\mathrm{T}_{3}\right)$ & $11.17 *$ & 57.74 & average $\times$ good \\
\hline & SEL-10-2 $\left(\mathrm{L}_{8}\right) \times$ California Wonder $\left(\mathrm{T}_{2}\right)$ & $10.26^{*}$ & 39.08 & average $\times$ poor \\
\hline & Kashmir Sel-1 $\left(\mathrm{L}_{2}\right) \times$ Solan Bharpur $\left(\mathrm{T}_{1}\right)$ & $7.45^{*}$ & 52.12 & average $\times$ average \\
\hline \multirow[t]{3}{*}{ Marketable fruit yield/plant (kg) } & ARCH-19 $\left(\mathrm{L}_{1}\right) \times$ Solan Bharpur $\left(\mathrm{T}_{1}\right)$ & $0.32 *$ & 1.40 & average $\times$ good \\
\hline & DARL-10 $\left(\mathrm{L}_{9}\right) \times$ California Wonder $\left(\mathrm{T}_{2}\right)$ & $0.28 *$ & 1.32 & good $\times$ poor \\
\hline & $\operatorname{UHFSP}(\mathrm{Y})-11\left(\mathrm{~L}_{11}\right) \times$ Yolo Wonder $\left(\mathrm{T}_{3}\right)$ & $0.27 *$ & 1.28 & good $\times$ poor \\
\hline \multirow[t]{3}{*}{ Plant height (cm) } & Kashmir Sel-1 $\left(\mathrm{L}_{2}\right) \times$ Solan Bharpur $\left(\mathrm{T}_{1}\right)$ & $44.28 *$ & 206.33 & good $\times$ poor \\
\hline & $\operatorname{UHFSP}(\mathrm{Y})-11\left(\mathrm{~L}_{11}\right) \times$ Solan Bharpur $\left(\mathrm{T}_{1}\right)$ & $24.85^{*}$ & 170.67 & good $\times$ poor \\
\hline & ARCH-9 $\left(\mathrm{L}_{6}\right) \times$ Yolo Wonder $\left(\mathrm{T}_{3}\right)$ & $24.27^{*}$ & 135.00 & poor $\times$ poor \\
\hline \multirow[t]{2}{*}{ Harvest duration (days) } & $\operatorname{UHFSP}(\mathrm{Y})-11\left(\mathrm{~L}_{11}\right) \times$ Yolo Wonder $\left(\mathrm{T}_{3}\right)$ & $19.30^{*}$ & 112.33 & average $\times$ average \\
\hline & DARL-01 $\left(\mathrm{L}_{5}\right) \times$ California Wonder $\left(\mathrm{T}_{2}\right)$ & $16.86^{*}$ & 124.33 & average $\times$ average \\
\hline \multirow[t]{3}{*}{ Ascorbic acid content (mg/100g) } & DARL-10 $\left(\mathrm{L}_{9}\right) \times$ California Wonder $\left(\mathrm{T}_{2}\right)$ & $26.42 *$ & 115.33 & poor $\times$ good \\
\hline & ARCH-19 $\left(\mathrm{L}_{1}\right) \times$ Solan Bharpur $\left(\mathrm{T}_{1}\right)$ & $26.12 *$ & 115.67 & average $\times$ average \\
\hline & $\operatorname{UHFSP}(\mathrm{Y})-11\left(\mathrm{~L}_{11}\right) \times$ Yolo Wonder $\left(\mathrm{T}_{3}\right)$ & $17.95^{*}$ & 108.11 & good $\times$ poor \\
\hline \multirow[t]{3}{*}{ Capsaicin content $(\%)$} & DARL-10 $\left(\mathrm{L}_{9}\right) \times$ California Wonder $\left(\mathrm{T}_{2}\right)$ & $-0.06^{*}$ & 0.09 & poor $\times$ average \\
\hline & ACC-16 $\left(\mathrm{L}_{4}\right) \times$ California Wonder $\left(\mathrm{T}_{2}\right)$ & $-0.05 *$ & 0.18 & poor $\times$ average \\
\hline & $\operatorname{UHFSP}(\mathrm{Y})-11\left(\mathrm{~L}_{11}\right) \times$ Solan Bharpur $\left(\mathrm{T}_{1}\right)$ & $-0.05^{*}$ & 0.06 & good $\times$ average \\
\hline
\end{tabular}

fects (Table 5). Good general combiners for number of marketable fruits per plant, having significant positive general combining ability effects, were Kashmir Sel-1 $\left(\mathrm{L}_{2}\right)$ followed by lines, DARL-10 $\left(\mathrm{L}_{9}\right)$, UHFSP(Y)-11 $\left(\mathrm{L}_{11}\right)$ and DARL-01 $\left(\mathrm{L}_{5}\right)$. For pericarp thickness, the lines viz., PRC-1 $\left(\mathrm{L}_{3}\right), \mathrm{ARCH}$ -19 $\left(\mathrm{L}_{1}\right)$, Kashmir Sel-1 $\left(\mathrm{L}_{2}\right)$, Kandaghat Sel-9 $\left(\mathrm{L}_{7}\right)$ and $\mathrm{LC}\left(\mathrm{L}_{10}\right)$ were good general combiners. The lines, SEL-10-2 $\left(\mathrm{L}_{8}\right)$ and ARCH-19 $\left(\mathrm{L}_{1}\right)$ were good general combiners for fruit length showing signifi- cant positive general combining ability effects. The lines, $\operatorname{LC}\left(\mathrm{L}_{10}\right)$ and $\operatorname{UHFSP}(\mathrm{Y})-11\left(\mathrm{~L}_{11}\right)$ exhibited significant positive general combining ability effect for average fruit weight. With regard to marketable fruit yield per plant, the lines viz. Kashmir Sel-1 $\left(\mathrm{L}_{2}\right)$, DARL-10 $\left(\mathrm{L}_{9}\right)$, $\operatorname{UHFSP}(\mathrm{Y})-11\left(\mathrm{~L}_{11}\right)$ and $\mathrm{LC}$ $\left(\mathrm{L}_{10}\right)$ were the good general combiners as reflected by their significant positive general combining ability effects. The lines viz. Kashmir Sel-1 ( $\left.\mathrm{L}_{2}\right)$, UHFSP(Y)-11 (L $\left.\mathrm{L}_{11}\right)$, ARCH-19 (L $\left.\mathrm{L}_{1}\right)$ and Kandaghat 
Sel-9 $\left(\mathrm{L}_{7}\right)$ exhibited the highest general combining ability effects in positive direction for plant height, indicating good general combining ability. For harvest duration, the line Kashmir Sel-1 $\left(\mathrm{L}_{2}\right)$ depicted significant positive general combining ability effect, thereby showing its good general combining ability. Good general combiners exhibiting significant positive general combining ability effects for ascorbic acid content were Kandaghat Sel-9 $\left(\mathrm{L}_{7}\right)$, Kashmir Sel-1 $\left(\mathrm{L}_{2}\right)$, UHFSP(Y)-11 $\left(\mathrm{L}_{11}\right)$ and $\mathrm{LC}\left(\mathrm{L}_{10}\right)$. For capsaicin content, all the lines except PRC-1 $\left(\mathrm{L}_{3}\right)$, ARCH-9 $\left(\mathrm{L}_{6}\right)$, SEL-10-2 $\left(\mathrm{L}_{8}\right)$ and DARL-10 (L 9$)$, were good general combiners depicting significant negative general combining ability effects. Amongst the testers, Solan Bharpur $\left(T_{1}\right)$ was the best for further use in crossing programme as it proved to be the best general combiner for most of the important traits viz. marketable fruit yield per plant, number of marketable fruits per plant, days to 50 per cent flowering, days to first harvest and pericarp thickness (Table 6). Different parental lines and testers expressing significant positive and negative and also significant and non-significant GCA effects in respect of yield and component traits in bell pepper have also been reported earlier by Geleta and Labuschagne 2006, Gomide et al. 2008, Kamble et al. 2009 and Sood and Kumar 2010.

The crosses with significant desirable SCA effects for various traits along with mean performance and GCA effects of parents involved in the crosses are listed in Table 7. The crosses, ARCH-19 $\left(\mathrm{L}_{1}\right) \times$ Solan Bharpur $\left(\mathrm{T}_{1}\right)$, DARL-10 $\left(\mathrm{L}_{9}\right) \times$ California Wonder $\left(\mathrm{T}_{2}\right)$ and $\operatorname{UHFSP}(\mathrm{Y})-11\left(\mathrm{~L}_{11}\right) \times$ Yolo Wonder $\left(\mathrm{T}_{3}\right)$ exhibited significant and desirable specific combining ability effects in respect of marketable fruit yield per plant and number of marketable fruits per plant. The cross combinations viz. DARL-10 (L $\left.\mathrm{L}_{9}\right)$ $\times$ California Wonder $\left(\mathrm{T}_{2}\right)$, DARL-01 $\left(\mathrm{L}_{5}\right) \times$ California Wonder $\left(\mathrm{T}_{2}\right)$, LC $\left(\mathrm{L}_{10}\right) \times$ Solan Bharpur $\left(\mathrm{T}_{1}\right)$ and Kandaghat Sel-9 $\left(\mathrm{L}_{7}\right) \times$ Solan Bharpur $\left(\mathrm{T}_{1}\right)$ were top five crosses showing significant negative SCA for earliness. The estimation of specific combining ability effects for pericarp thickness revealed that cross combinations viz. UHFSP(Y)-11 $\left(\mathrm{L}_{11}\right) \times$ Yolo Wonder $\left(\mathrm{T}_{3}\right)$, DARL-10 $\left(\mathrm{L}_{9}\right) \times$ California Wonder $\left(\mathrm{T}_{2}\right), \mathrm{ARCH}-$ $19\left(\mathrm{~L}_{1}\right) \times$ Solan Bharpur $\left(\mathrm{T}_{1}\right)$, Kandaghat Sel-9 $\left(\mathrm{L}_{7}\right) \times$ Solan Bharpur $\left(\mathrm{T}_{1}\right)$ and Kandaghat Sel-9 $\left(\mathrm{L}_{7}\right) \times$ California Wonder $\left(\mathrm{T}_{2}\right)$ were top three good specific combinations. Lobe per fruit is an important horticultural trait in bell pepper from consumers' point of view. The only cross combination expressing significant desirable specific combining ability effects for this trait was $\operatorname{UHFSP}(\mathrm{Y})-11\left(\mathrm{~L}_{11}\right) \times$ Yolo Wonder $\left(\mathrm{T}_{3}\right)$. For fruit length the crosses Kandaghat Sel-9 $\left(\mathrm{L}_{7}\right) \times$ Yolo Wonder $\left(\mathrm{T}_{3}\right)$, ACC-16 $\left(\mathrm{L}_{4}\right) \times$ Solan Bharpur $\left(\mathrm{T}_{1}\right)$ and DARL-10 $\left(\mathrm{L}_{9}\right) \times$ California Wonder $\left(\mathrm{T}_{2}\right)$ showed significant positive specific combining ability effects indicating their good specific combining ability while, Only one of the crosses viz. UHFSP $(\mathrm{Y})-11\left(\mathrm{~L}_{11}\right) \times \mathrm{Yo}-$ lo Wonder $\left(\mathrm{T}_{3}\right)$ expressed significant positive specific combining ability effect for fruit width. The five cross combinations viz. DARL-10 $\left(\mathrm{L}_{9}\right) \times$ Yolo Wonder $\left(\mathrm{T}_{3}\right)$, SEL-10-2 $\left(\mathrm{L}_{8}\right) \times$ California Wonder $\left(\mathrm{T}_{2}\right)$, Kashmir Sel$1\left(\mathrm{~L}_{2}\right) \times$ Solan Bharpur $\left(\mathrm{T}_{1}\right) \operatorname{UHFSP}(\mathrm{Y})-11\left(\mathrm{~L}_{11}\right) \times$ Yolo Wonder $\left(\mathrm{T}_{3}\right)$ and ARCH-19 $\left(\mathrm{L}_{1}\right) \times$ Solan Bharpur $\left(\mathrm{T}_{1}\right)$ expressed significant desirable specific combining ability effects for average fruit weight. The crosses viz. Kashmir Sel-1 $\left(\mathrm{L}_{2}\right) \times$ Solan Bharpur $\left(\mathrm{T}_{1}\right), \operatorname{UHFSP}(\mathrm{Y})-$ $11\left(\mathrm{~L}_{11}\right) \times$ Solan Bharpur $\left(\mathrm{T}_{1}\right)$, ARCH-9 $\left(\mathrm{L}_{6}\right) \times$ Yolo Wonder $\left(\mathrm{T}_{3}\right)$ and ACC-16 $\left(\mathrm{L}_{4}\right) \times$ Yolo Wonder $\left(\mathrm{T}_{3}\right)$ were the top three specific combiners for plant height. For harvest duration significant positive specific combining ability effects were observed for two crosses viz. $\operatorname{UHFSP}(\mathrm{Y})-11 \quad\left(\mathrm{~L}_{11}\right) \times$ Yolo Wonder $\left(\mathrm{T}_{3}\right)$ and DARL-01 $\left(\mathrm{L}_{5}\right) \times$ California Wonder $\left(\mathrm{T}_{2}\right)$. Majority of the cross combinations exhibiting desirable specific combining ability effects, involved atleast one of the parents as good or average general combiner. Similar results have also been obtained by earlier researchers viz. Sood and Kaul 2006; Sood and Kumar 2010 and Rao PG et al. 2016. In some of the crosses, significant specific combining ability effects were observed but, they had both the parents as poor general combiners. These findings corroborate the observations of Pandey et al. 2002; Sood and Kumar 2010 and Pandey et al. 2012, who have also observed that the superior hybrids need not necessarily involve parents showing good general combining ability effects only. Usually the high estimates of specific combining ability effects are obtained from crosses involving diverse parents. Sometimes, due to specific interaction effects (most likely complementary), poor $\times$ poor crosses may prove better than $\operatorname{good} \times \operatorname{good}$ and good $\times$ poor combinations. The crosses involving both the parents as poor combiners showing high specific combining ability effects must be due to intra and inter allelic interactions and these crosses can be used for commercial exploitation of hybrid vigour as the non-additive genes seem to play a greater role.

\section{Conclusion}

On the basis of the estimates of general combining ability the cross combinations viz. Kashmir Sel-1 (L2), DARL-10 (L9) and UHFSP(Y)-11 (L11) were the most promising for marketable fruit yield per plant. These cross combinations can be further evaluated for development of superior genotypes and exploiting hybrid vigour in bell pepper. The cross combinations viz. ARCH-19 (L1) $\times$ Solan Bharpur (T1), DARL-10 (L9) × California Wonder (T2), UHFSP(Y)-11 (L11) × Yolo Wonder (T3), DARL01 (L5) $\times$ California Wonder (T2) and Kandaghat Sel-9 (L7) $\times$ Solan Bharpur (T1) were observed to be the top five cross combinations with significant 
SCA effects for marketable fruit yield per plant under protected environment. Thus the present study pointed out that the above promising crosses can be commercially exploited for development of early and high yielding hybrids of bell pepper suitable for protected cultivation in mid hills of Western Himalayas.

\section{REFERENCES}

Abebe, T., Alamerew, S. and Tulu, L. (2017). Genetic variability, heritability and genetic advance for yield and its related traits in rainfed lowland rice (Oryza sativa L.) genotypes at Fogera and Pawe, Ethiopia. Adv. Crop Sc. and Tech. 5(2): 272

Afroza, B., Khan, S.H., Mushtaq, F., Hussain, K. and Nabi. (2013). Variability and correlation studies in sweet pepper (Capsicum annuum L.). Prog. Hort. 45: 209-213

Burton, G.W. and DeVane, E.H.D. (1953). Estimating heritability in tall fescue (Fescue arundinaceae) from replicated clonal material. Agron. J. 54: 478-481

Geleta, L.F. and Labuschagne, M.T. (2006). Combining ability and heritability for vitamin $\mathrm{C}$ and total soluble solids in pepper (Capsicum annuum L.). J. Sci. Food Agric. 86: 1317-1320

Gomide, M.L., Maluf W.R. and Gomes, L.A.A. (2008). Combining ability among lines of sweet pepper (Capsicum annum L.) Cienc. Agrotec. 32: 740-748

Islam, S. and Singh R.V. (2006). Variability, heritability and genetic advance studies in sweet pepper (Capsicum annuum L. var. grossum Sendt.). Indian J. of Pl. Gen. Rsc. 19: 203-205

Johnson, H.W., Robinson, H.F. and Comstock, R.E. (1955). Estimates of genetic and environmental variability in soyabean. Agron. J. 47: 314-318

Kadambavanasundaram, M. (1980). Heterotic system in cultivated species of Gossypium. An appraisal (Abst). Genetic and crop improvement of heterotic systems. Pre-congress scientific meeting of XV International Congress of Genetics, TNAU, Coimbatore, pp. 20.

Kamble, C., Mulge, R. and Madalageri, M.B. (2009). Combining ability for earliness and productivity in sweet pepper (Capsicum annuum L.). Karnataka J. of Agri. Sc. 22: 151-154

Kempthrone, O. (1957). An Introduction to Genetic Statistics. John Wiley and Sons, New York, London.
Kumar, R. and Kumar, S. (2017). Usefulness of combining ability and gene action studies for parthenocarpic gynoecious hybrid development in cucumber. $J$. of Hill Agri. 8: 158-165

Kumari, S. (2013). Genetic variability studies in bell pepper (Capsicum annuum L.). The Asian J. of Hort. 8: 280-284

Pandey, V., Ahmed Z. and Kumar, N. (2002). Heterosis and combining ability in diallel crosses of sweet pepper (Capsicum annuum L.). Veg. Sc. 29: 66-67

Pandey, V., Chura, A., Pandey, H.K., Meena, H.S., Arya, M. and Ahmed, Z. (2012). Diallel analysis for yield and yield attributing traits in capsicum (Capsicum annuum L. var grossum Sendt). Veg. Sc. 39: 136-139

Ranganna S. (1979). Manuals of Analysis of Fruits and Vegetable Products. Tata McGraw Hill Book Company, New Delhi.

Rao, P.G., Reddy, K.M., Naresh, P., Chalapathi, V.V. and Indiresh, K.M. (2016). Combining ability and standard heterosis in bell pepper (Capsicum annuum L.) for yield and yield attributing traits. Green Farming Int. J. 7 (5): 1024-1028

Rattan, P. and Chadha, S, (2009). Gene action studies for yield and its contributing characters. Biological Forum- An Inter. J. 1(2): 8-10.

Rohini, N., Lakshmanan, V., Saraladevi, D., Amalraj, J.J and Govindaraju, P. (2017). Assessment of combining ability for yield and quality components in hot pepper (Capsicum annuum L.). Spanish J. of Agric. Res. 15(2): 1-12

Sahoo, T.R. and Singh, D.K. (2017). Estimates of combining ability for development of $\mathrm{F}_{1}$ hybrids in cucumber. J. of Hill Agri. 8(3): 261-269

Singh, D.P., Anand, N. and Deshpande, A.A. (1993). Improvement of bell pepper. In: Advances in Horticulture. Veg. Crops. 5: 87-104

Sood, S. and Kaul, S. (2006). Combining ability in bell pepper. Veg. Sc. 33: 73-75

Sood, S. and Kumar, N. (2010). Combining ability and gene action for phenological, structural and fruit yield traits in bell pepper (Capsicum annuum L. var grossum Sendt.) under sub temperate zone of North western Himalayas. Advances in Hort. Sc. 24: 122-128

Sood, S., Sharma, A. and Chaudhary, D.R. (2007). Genetic architecture and trait relationship in bell pepper under sub-temperate conditions of north western Himalayas. Indian J. of Hort. 64: 169-174 